\title{
COMPARISON OF DIFFERENT CHEST COMPRESSION POSITIONS FOR USE WHILE WEARING CBRN-PPE: A RANDOMIZED CROSSOVER SIMULATION TRIAL
}

\author{
Marek Malysz ${ }^{1,2}{ }^{\infty}$, Milosz Jaroslaw Jaguszewski ${ }^{3}$, Lukasz Szarpak ${ }^{1,4,5}{ }^{\circ}$, Dominika Telecka-Gadek ${ }^{1,6}{ }^{\infty}$, \\ Karol Bielski ${ }^{1,2}{ }^{(0}$, Agata Dabrowska ${ }^{1,7}{ }^{(0}$, Jacek Smereka ${ }^{1,8}{ }^{\infty}$, Krzysztof Jerzy Filipiak ${ }^{9}$ \\ ${ }^{1}$ Polish Society of Disaster Medicine, Warsaw, Poland \\ ${ }^{2}$ Voivodeship Emergency Ambulance Service and Sanitary Transport "MEDITRANS", Warsaw, Poland \\ ${ }^{3}$ First Department of Cardiology, Medical University of Gdansk, Poland \\ ${ }^{4}$ Bialystok Oncology Center, Bialystok, Poland \\ ${ }^{5}$ Maria Sklodowska-Curie Medical Academy, Warsaw, Poland \\ ${ }^{6}$ Department of Emergency Medicine, Medical University of Warsaw, Poland \\ ${ }^{7}$ Department of Emergency Medical Service, Poznan University of Medical Sciences, Poznan, Poland \\ ${ }^{8}$ Department of Emergency Medical Service, Wroclaw Medical University, Wroclaw, Poland \\ ${ }^{9}$ First Chair and Department of Cardiology, Medical University of Warsaw, Poland
}

\section{ABSTRACT}

INTRODUCTION: The prevailing COVID-19 pandemic forces paramedics to take medical rescue operations using personal protective equipment (PPE) for aerosol-generating procedures (AGP). The use of PPE-AGP may reduce the effectiveness of the procedures performed, including airway management, intravascular access, or chest compression.

The goal of the current study was to compare the quality by which a chest compression during simulated COVID-19 resuscitation while wearing PPE-AGP. A secondary goal was to assess provider preferences with standard versus OHD chest compression methods while wearing PPE-AGP.

METHODS: This is a randomized cross-over single-blinded study involving 37 paramedics performing 2-min continuous chest compression using two methods: the standard chest compression (CC) method during which the rescuer takes a position to the side of the victim (STD) and over-the-head position (OHD). During cardiopulmonary resuscitation, study participants wore Class C PPE-AGP. Both the order of study participants and compression methods were random. The results were blinded before statistical analysis. The compression rate per minute (CPM), CC depth as well as full chest recoil were measured. The analysis was undertaken using STATISTICA (V13.3EN).

RESULTS: Mean chest compression depth using distinct CC methods varied and amounted to $42 \pm 2 \mathrm{~mm}$ for STD vs. $46 \pm 4 \mathrm{~mm}$ for OHD $(p<0.001)$. Chest compressions based on the OHD method were associated with a lower frequency of chest compressions (107 \pm 7 CPM) compared with STD $(114.5 \pm 8 ; p<0.001)$. A higher percentage of full chest recoil was observed in the case of STD ( $42 \pm 6 \%)$ than in the case of OHD (34 $\pm 10 \%)$.

CONCLUSIONS: Based on the current simulation trial, it is impossible to clearly determine which method (STD vs. OHD) is more effective in resuscitation with PPE-AGP. Paramedics wearing PPE-AGP achieved better chest compression depth for OHD compared to the STD, however, OHD resuscitation causes a lower degree of full chest relaxation. A further well-designed clinical study looking at efficacy, safety, and outcomes is needed to confirm current results. 
KEY WORDS: chest compression, cardiopulmonary resuscitation, quality, position, personal protective equipment, CBRN, COVID-19, SARS-CoV-2, pandemic, medical simulation, manikin

Disaster Emerg Med J 2020; 5(3): 127-133

\section{INTRODUCTION}

The outbreak of coronavirus disease 2019 (COVID-19) has become a primary challenging public health issue [1]. On March 11, 2020, the World Health Organization declared that the pandemic of COVID-19 had become a public health emergency of global concern. As of September 2, 2020 COVID-19 has been reported with a total of 25.835 .301 confirmed cases and over 858.661 deaths. New coronavirus SARS-CoV-2 is mainly transmitted through 1) Direct exposure with cough, sneeze, and droplet inhalation within a range of about 1.8 meters; and 2) Contact transmission through contact with oral, nasal, and eye mucous membranes [2, 3]. Current personal protective equipment (PPE) and infection control guidelines from the WHO is based on the assumption that the primary mechanism of transmission is direct and indirect droplet spread. In this situation, paramedics working in the pre-hospital setting should treat any patient as potentially infected with the SARS-CoV-2 virus $[4,5]$. The WHO advises that airborne transmission can occur, but only when aerosol-generating procedures (AGP) are performed [6]. AGPs are those that have the potential to generate aerosols and droplets that can spread respiratory pathogens. In this context, cardiopulmonary resuscitation (CPR) is a complex procedure that is particularly dangerous for medical personnel. As the study by Borkowska et al. examining the effectiveness of cardiopulmonary resuscitation during the COVID-19 pandemic, the return of spontaneous circulation (ROSC) in prehospital setting was observed only in $9.4 \%$ of resuscitated patients [7]. In turn, Baldi et al. indicating the OHCA issues in Italy indicate that during the first 40 days of the COVID-19 outbreak (February 21 through March $31,2020)$ with those that occurred during the same period in 2019 and indicate a 58\% increase rate of OHCA in 2020 [8]. Low patient survival may be caused by both the negative consequences of SARSCoV-2 infection, as well as the reduced effectiveness of the procedure performed by Emergency Medical Service personnel wearing PPE-AGP.

The goal of the current study was to compare the quality by which a chest compression during simulated COVID-19 resuscitation while wearing PPE-AGP. A secondary goal was to assess provider preferences with standard versus OHD chest compression methods while wearing PPE-AGP.

\section{METHODS}

\section{Study Population and Setting}

The study population consisted of paramedics participating in the training of Advanced Cardiovascular Life Support (ACLS) conducted according to the AHA guidelines [9]. Inclusion criteria were: a) consent to participate in the study, b) active paramedic status, c) minimum one year of experience in EMS teams. Exclusion criteria were: a) refusal to consent, b) presence of medical concerns precluding the ability to participate, including pregnancy or asthma, c) symptoms or suspicion of viral infection.

\section{Study Design}

A prospective, randomized, crossover single-blinded simulation trial was conducted. The protocol of this trial was approved by the Institutional Review Board of the Polish Society of Disaster Medicine (No. 22.01.20.IRB). The study was conducted according to the Consolidated Standards of Reporting Trials (CONSORT) statement (Suppl. 1).

Before beginning the simulation portion of the study, all participants were asked to complete a short questionnaire concerning previous experiences with clinical resuscitation as well as PPE-AGP using. Additionally, all participants participated in a 60-minute training session reminding the principles of cardiopulmonary resuscitation following the guidelines of the American Heart Association (AHA) [9], as well as the demonstration of the correct donning of the PPE-AGP suit and performing chest compression using the STD and OHD method (Fig. 1).

After the demonstration session, participants were randomized to STD or OHD while wearing a Tychem F Level-C (DuPont, Wilmington, USA) suit, airway protection N95 respirator (3M Poland, Kajetany, Poland), face shield (3M Poland, Kajetany, Poland) including double nitrile gloves (MedaSEPT ${ }^{\circledR}$, Poznan, Poland).

The participants were then asked to perform a 2-minute cycle of continuous chest compression (CCC) using one method, then had a 30-minute 
(A)

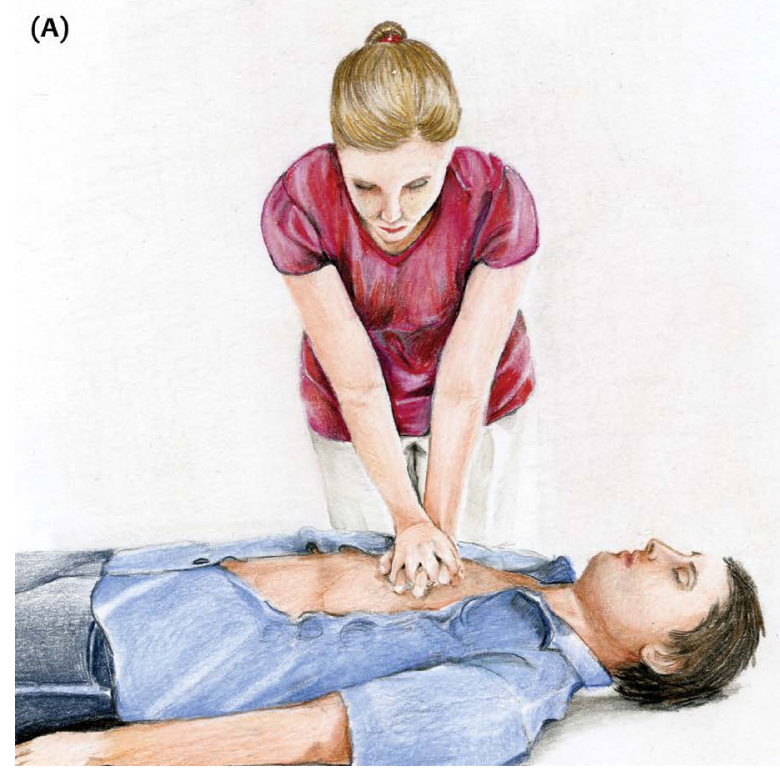

(B)

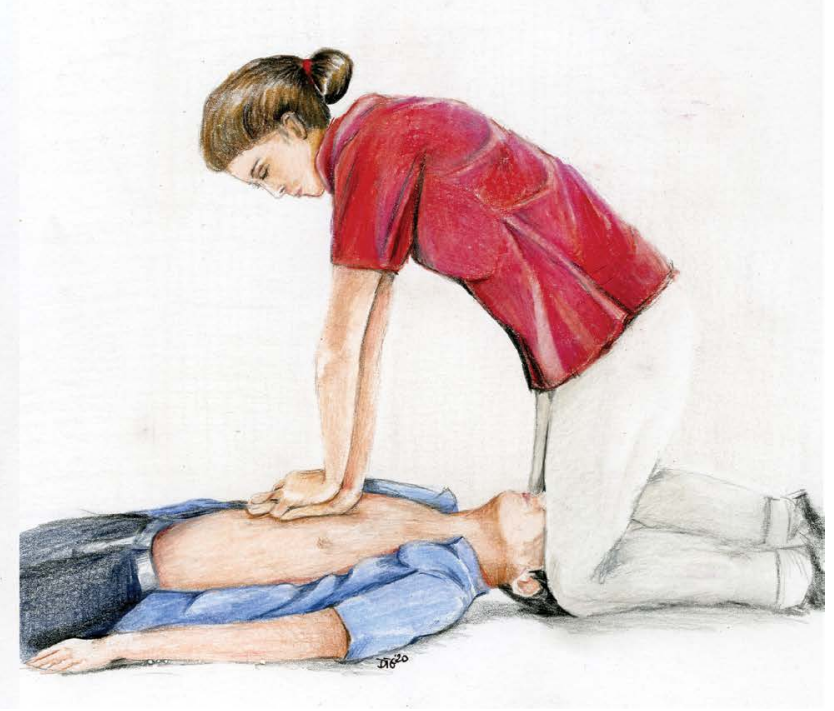

FIGURE 1. Chest compression methods used in the trial: (A) Standard position; (B) Over-the-head position

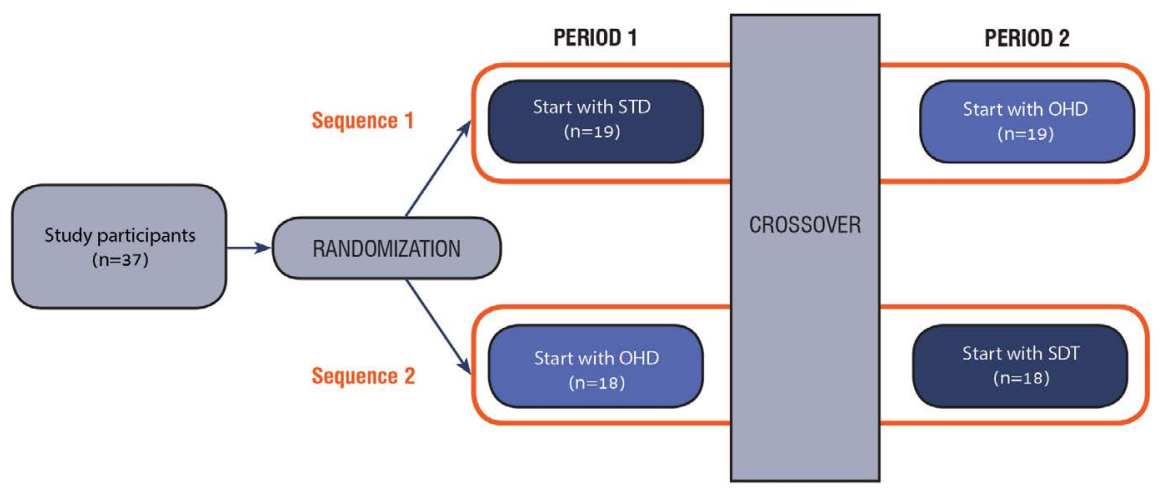

FIGURE 2. Randomization flow chart

break, and then performed chest compression using another method. The detailed procedure of trial randomization is presented in Figure 2.

All chest compressions were performed on a SimMan 3G (Laerdal Inc., Stavanger, Norway) adult simulator. The simulator was placed on a flat surface to simulate sudden cardiac arrest at home.

After performing chest compression both methods, each participant completed the post-intervention questionnaire regarding perceptions of ease of chest compression while wearing PPE-AGP.

\section{Outcomes and Data Collection}

The primary outcome measure was the quality of chest compression, which consisted of the following parameters: chest compression rate, chest compression depth as well as chest recoil. The values indicated in the AHA guidelines [9] were used as reference values. Accordance with those guidelines, chest compression rate should be between 100 and 120 compressions per minute (CPM), the depth of chest compressions should be 5 to $6 \mathrm{~cm}$, and after each compression should be followed by full relaxation of the chest.

The chest compression quality data was recorded in real-time using the simulator control software - Laerdal Learning Application (LLEAP software, v.7.1.0; Laerdal Inc, Stavanger, Norway).

De-identified participants' data were entered into Macintosh Excel Database (Microsoft Excel for MAC 2020, v.16.40; Microsoft Inc., Redmond, WA, USA). Data were correlated with questionnaire response data by the use of pre-assigned participant numbers. 


Table 1. Summary of study results
\begin{tabular}{|l|c|c|c|c|}
\hline \multicolumn{1}{|c|}{ Compression parameter } & STD & OHD & MD / OR $(95 \% C l)$ & p-value \\
\hline CC depth $(m m)$ & $42( \pm 2)$ & $46( \pm 4)$ & MD $=-4.00(-5.44,-2.56)$ & $<0.001$ \\
\hline CC rate (CPM) & $114.5( \pm 8)$ & $107( \pm 7)$ & $\mathrm{MD}=7.50(4.07,10.93)$ & $<0.001$ \\
\hline Chest recoil (\%) & $42( \pm 6)$ & $34( \pm 10)$ & $\mathrm{MD}=8.00(4.24,11.76)$ & $<0.001$ \\
\hline Correct hand placement (\%) & $96.5( \pm 2.5)$ & $96( \pm 4)$ & $\mathrm{MD}=0.50(-1.02,2.02)$ & 0.52 \\
\hline Preferences $(\%)$ & $16 / 37(43.2 \%)$ & $21 / 37(56.8 \%)$ & $\mathrm{OR}=0.58(0.23,1.46)$ & 0.25 \\
\hline
\end{tabular}

CC — Chest compression; CPM — Compressions per minute; STD — Standard position; OHD — Over-the-head position; MD — Mean difference; OR — Odds ratio; Cl — Confidence interval

\section{Statistical analysis and Sample Size Calculations}

Sample size calculations were performed based on a two-sided paired t-test assuming $80 \%$ power and a significance level of 0.05. Assumptions for expected results were based upon the work of Malysz et al. [10]. Those calculations indicated that a sample size of 32 participants would be required to power the trial adequately to detect a difference of 5 millimeters between the two modalities. To ensure a safety margin, we recruited 37 participants in this study.

All calculations were done with the STATISTICA software package (v.13.3EN; Tibco Inc, Tulsa, OK, USA). For statistical analysis, the data was blinded. Continuous features were summarized with means and standard deviations. Categorical features were summarized with frequency counts and percentages. The Kolmogorov-Smirnov test was applied to test data for normality. Fisher Exact Tests were used to compare resident survey responses. We compared qualitative variables by Fisher exact test and Kruskal-Wallis test. Continuous data, including the time for the successful intravascular access, were analyzed using analysis of variance (ANOVA) testing. All tests were two-sided and $p$ values less than 0.05 were considered statistically significant.

\section{RESULTS}

The study involved 37 paramedics whose mean age was $31 \pm 7.5$ years and mean work experience in EMS was $6.7 \pm 4.5$ years. All participants in the study declared their experience in the field of CPR in clinical settings. None of the study participants had experience with chest compression wearing PPEAGP before study entry.

A detailed summary of the results of chest compression parameters is presented in Table 1. Mean chest compression depth using distinct CC methods varied and amounted to $42 \pm 2 \mathrm{~mm}$ for STD vs. $46 \pm 4 \mathrm{~mm}$ for OHD $(p<0.001)$. Chest com- pressions based on the OHD method were associated with a lower frequency of chest compressions $(107 \pm 7$ 7PM) compared with STD (114.5 \pm 8 ; $p<0.001)$. A higher percentage of full chest recoil was observed in the case of STD $(42 \pm 6 \%)$ than in the case of OHD (34 $\pm 10 \%)$. This difference was statistically significant $(p<0.001)$. The differences incorrect hand placement between STD and OHD were not statistically significant.

Most people (58.8\%) indicated OHD as the preferred method during true CPR. The remaining people indicated the STD method as the preferred method.

\section{DISCUSSION}

The aim of the study was to compare two techniques of chest compression (STD vs. OHD) performed by paramedics wearing PPE-AGP. The prevailing COVID-19 pandemic causes that in the case of OHCA paramedics should perform CPR in protective suits. To the authors' knowledge, this is the first STD vs. OHD under the conditions of use personal protective equipment against aerosol-generating procedures.

Performing medical procedures when healthcare workers are wearing PPE-AGP may reduce the effectiveness of the procedures performed by extending the duration of these procedures as well as reducing the effectiveness of individual attempts to perform the procedure. This applies to both obtaining intravascular access [11], advanced airway management [12], as well as the effectiveness of chest compression [13]. Chen et al. [14] indicated significant deterioration of $\mathrm{CC}$ performance in $\mathrm{HCW}$ s with the use of a level-C personal protective equipment, which may be a disadvantage for enhancing the survival of cardiac arrest. In turn, a study by Donoghue et al. shows that during a clinically appropriate 2-minute period, neither CC quality nor self-reported fatigue worsened to a significant degree in providers wearing PPE [15]. Donghue also indicates that that 
Pediatric Basic Life Support recommendations for CC providers to switch every 2 minutes need not be altered with PPE use. The research of Malysz et al. [13] indicates that in the case of adult resuscitation, the use of PPE reduces the quality of chest compression, including a reduction in the depth of chest compressions and the correctness of chest relaxation with time. The differences between these two studies can be explained by the fact that resuscitation was carried out concerning the various simulators: an adult and a child. In the study by Malysz et al. an adult simulator was used, whose cardiopulmonary resuscitation requires much more effort than the resuscitation of a child. On the other hand, Donghue indicates that the depth of chest compression did not decrease significantly over time, however, according to the analysis of the data obtained by him, chest compression was performed based on too shallow chest compressions, which were on average $2.2 \mathrm{~cm}$, while the guidelines of resuscitation for 5 -years old child recommend that the CC depth be between $1 / 3$ to $1 / 2$ of the anteroposterior diameter of the chest, corresponding to a depth of at least $5 \mathrm{~cm} \mathrm{[16].} \mathrm{Nerveless,} \mathrm{it} \mathrm{is} \mathrm{clear} \mathrm{that} \mathrm{the}$ returning of the spontaneous circulation (ROSC) in patients experiencing cardiac arrest is dependent on the quality of the CPR they receive. However, a number of investigations have demonstrated that rescuers develop immediate fatigue during CPR and the quality of CC declines rapidly after 1 to $3 \mathrm{~min}$ of CPR [17]. Abelairas-Gómez et al. showed that a simple strength training program has a significant impact on the quality of chest compressions and its maintenance over time [18]. The analysis of the performed examination showed a statistically significantly greater depth of chest compressions during compression performed by OHD. This may be due to the force applied to the chest being directed differently, thus exerting more pressure on the chest than is the case with an STD.

Another parameter, apart from the depth of chest compressions, influencing the quality of chest compression is the $\mathrm{CC}$ rate per minute. The chest compression rate was also associated with the return of spontaneous circulation $[19,20]$. CPR guidelines performer by AHA as well as ERC recommends that the CPR be greater than 100CPM, but not greater than 120CPM [21, 22]. Despite the existence of differences in the rate of chest compression despite the STD and OHD groups, these rates were within the recommended range. Despite subsequent editions of the CPR guidelines, there is still no consensus among scientists regarding the optimal rate of chest compression. As indicated Solevåg et al. [23] and other authors, a higher CC rate (i.e. over $120 / \mathrm{min}$ ) is also more fatiguing, which affects CC quality [24]. In turn, a study by Kilgannon et al. conducted in in-hospital cardiac arrest adult patients, a chest compression rate of 121-140CPM had the highest odds ratio of ROSC [25]. In the case of OHCA, Idris et al. indicated that compression rates between 100 and 120CPM were associated with the greatest survival to hospital discharge [19].

In this study, the continuous chest compression technique was selected. According to the ERC and AHA guidelines, efforts should be made to minimize interruptions in chest compressions. Moreover, as evidenced by Ewy et al. [26] research, CCC compared with 30:2 compressions-to-ventilations cardiopulmonary resuscitation improved neurological outcome. Wang et al. in a prospective, randomized animal study in the first 12 minutes of CPR, continuous compressions could maintain relatively better coronary perfusion pressure, $\mathrm{PaO}_{2}$, and global ventilation/perfusion values than 30:2 cardiopulmonary resuscitation [27]. The above advantage of continuous chest compressions over 30:2 resuscitation was also confirmed in other studies [28-30]. However, as the study by Liu et al. shows, chest compression quality decreased significantly faster when performing CCC compared to 30:2 method [31]. Therefore, the person compressing the chest should be changed every two minutes or sooner.

Another parameter influencing the quality of chest compression is full chest recoil (FCR), which was independently associated with improved survival and favorable neurologic outcome at hospital discharge after adult OHCA [32]. In our study, the correctness of chest relaxation in both STD and OHD was insufficient, and in the case of OHD, a statistically significant reduction in the correctness of FCR was observed. This may be because paramedics tend to lean excessively on the patient's chest in over-the-head resuscitation.

The above-mentioned and described parameters of chest compression, such as the depth of chest compressions, the frequency of compression or the correctness of chest relaxation are extremely important and affect the quality of the compression and thus the effectiveness of the entire CPR, however, they should not be treated individually - but try to perform CPR based on all parameters of chest compression at the same time $[33,34]$. 


\section{Limitations and Strengths}

Limitations of the medical simulation trials have been previously described [13]. In addition, only a group of paramedics participated in the study, however, it was a deliberate action dictated by the fact that paramedics are the first line of contact with OHCA patients and are relatively often forced to undertake resuscitation measures in pre-hospital conditions, and in the time of the prevailing COVID pandemic -19, these procedures should be performed in PPE-AGP.

The study also has strengths. To the authors' knowledge, this is the first study to evaluate STD vs. OHD chest compression methods by paramedics while wearing PPE-AGP. Another strong point of the study is its randomized cross-over nature and the fact that it was a single-blinded study.

\section{CONCLUSIONS}

Based on the current simulation trial, it is impossible to determine which method (STD vs. OHD) is more effective in resuscitation with PPE-AGP. Paramedics wearing PPE-AGP achieved better chest compression depth for OHD compared to the STD, however, OHD resuscitation causes a lower degree of full chest relaxation. A further well-designed clinical study looking at efficacy, safety, and outcomes is needed to confirm current results.

\section{Supplement 1. CONSORT checklist}

Supplementary material related to this article can be found, in the online version, at: https://journals. viamedica.pl/disaster_and_emergency_medicine/article/view/DEMJ.a2020.0034\#supplementaryFiles

\section{Conflict of Interest}

All authors declare that they have no conflict of interest.

\section{Sources of funding}

None.

\section{Acknowledgement}

The authors would like to thank all paramedics who participated in this study. The study was supported by the ERC Research Net and by the Polish Society of Disaster Medicine.

\section{REFERENCES}

1. Dzieciatkowski T, Szarpak L, Filipiak KJ, et al. COVID-19 challenge for modern medicine. Cardiol J. 2020; 27(2): 175-183, doi: 10.5603/ CJ.a2020.0055, indexed in Pubmed: 32286679.
2. Xiao F, Sun J, Xu Y, et al. Infectious SARS-CoV-2 in Feces of Patient with Severe COVID-19. Emerg Infect Dis. 2020; 26(8): 1920-1922, doi: 10.3201/eid2608.200681, indexed in Pubmed: 32421494.

3. Vivanti AJ, Vauloup-Fellous $C$, Prevot $S$, et al. Transplacental transmission of SARS-CoV-2 infection. Nat Commun. 2020; 11(1): 3572, doi: 10.1038/s41467-020-17436-6, indexed in Pubmed: 32665677.

4. Smereka J, Szarpak L, Filipiak K. Modern medicine in COVID-19 era. Disaster and Emergency Medicine Journal. 2020, doi: 10.5603/demj. a2020.0012.

5. Pruc M, Golik D, Szarpak L, et al. COVID-19 in healthcare workers. Am J Emerg Med. 2020 [Epub ahead of print], doi: 10.1016/j. ajem.2020.05.017, indexed in Pubmed: 32414525.

6. Maji C. Modes of transmission of COVID-19 outbreak- a mathematical study. , doi: 10.1101/2020.05.16.20104315.

7. Gilliam S. Dual Defibrillation in Out-of-Hospital Cardiac Arrest: A Retrospective Cohort Analysis. The Journal of Emergency Medicine. 2016; 51(5): 616-617, doi: 10.1016/j.jemermed.2016.09.038.

8. Baldi E, Sechi GM, Mare C, et al. Lombardia CARe Researchers. Out-of-Hospital Cardiac Arrest during the Covid-19 Outbreak in Italy. N Engl J Med. 2020; 383(5): 496-498, doi: 10.1056/NEJMc2010418, indexed in Pubmed: 32348640.

9. Link MS, Berkow LC, Kudenchuk PJ, et al. Part 7: Adult Advanced Cardiovascular Life Support: 2015 American Heart Association Guidelines Update for Cardiopulmonary Resuscitation and Emergency Cardiovascular Care. Circulation. 2015; 132(18 Suppl 2): S444-S464, doi: 10.1161/CIR.0000000000000261, indexed in Pubmed: 26472995.

10. Malysz M, Bielski K, Smereka J, et al. Which technique of chest compression should we use wearing full personal protective equipment: a pilot data. Post N Med.; 2020.

11. Smereka J, Szarpak L, Filipiak KJ, et al. Which intravascular access should we use in patients with suspected/confirmed COVID-19? Resuscitation. 2020; 151: 8-9, doi: 10.1016/j.resuscitation.2020.04.014, indexed in Pubmed: 32304800.

12. Ludwin K, Bialka S, Czyzewski L, et al. Video laryngoscopy for endotracheal intubation of adult patients with suspected/ confirmed COVID-19. A systematic review and meta-analysis of randomized controlled trials. Disaster and Emergency Medicine Journal. 2020, doi: 10.5603/demj.a2020.0023.

13. Malysz M, Dabrowski M, Böttiger BW, et al. Resuscitation of the patient with suspected/confirmed COVID-19 when wearing personal protective equipment: A randomized multicenter crossover simulation trial. Cardiol J. 2020 [Epub ahead of print], doi: 10.5603/CJ.a2020.0068, indexed in Pubmed: 32419128.

14. Chen J, Lu KZ, Yi B, et al. Chest Compression With Personal Protective Equipment During Cardiopulmonary Resuscitation: A Randomized Crossover Simulation Study. Medicine (Baltimore). 2016; 95(14): e3262, doi: 10.1097/MD.0000000000003262, indexed in Pubmed: 27057878.

15. Donoghue AJ, Kou M, Good GL, et al. Best Pharmaceuticals for Children Act - Pediatric Trials Network. Impact of Personal Protective Equipment on Pediatric Cardiopulmonary Resuscitation Performance: A Controlled Trial. Pediatr Emerg Care. 2020; 36(6): 
267-273, doi: 10.1097/PEC.0000000000002109, indexed in Pubmed: 32483079.

16. de Caen AR, Berg MD, Chameides L, et al. Part 12: Pediatric Advanced Life Support: 2015 American Heart Association Guidelines Update for Cardiopulmonary Resuscitation and Emergency Cardiovascular Care (Reprint). Pediatrics. 2015; 136 Suppl 2(18 Suppl 2): S176-S195, doi: 10.1542/peds.2015-3373F, indexed in Pubmed: 26471384.

17. Ashton A, McCluskey $A$, Gwinnutt $C L$, et al. Effect of rescuer fatigue on performance of continuous external chest compressions over 3 min. Resuscitation. 2002; 55(2): 151-155, doi: 10.1016/503009572(02)00168-5, indexed in Pubmed: 12413752.

18. Abelairas-Gómez C, Barcala-Furelos R, Szarpak $\measuredangle$, et al. The effect of strength training on quality of prolonged basic cardiopulmonary resuscitation. Kardiol Pol. 2017; 75(1): 21-27, doi: 10.5603/KP.a2016.0165, indexed in Pubmed: 27878801.

19. Idris AH, Guffey D, Pepe PE, et al. Resuscitation Outcomes Consortium Investigators. Chest compression rates and survival following out-of-hospital cardiac arrest. Crit Care Med. 2015; 43(4): 840-848, doi: 10.1097/CCM.0000000000000824, indexed in Pubmed: 25565457.

20. Zhu Ni, Chen Qi, Jiang Z, et al. A meta-analysis of the resuscitative effects of mechanical and manual chest compression in out-of-hospital cardiac arrest patients. Crit Care. 2019; 23(1): 100, doi: 10.1186/ s13054-019-2389-6, indexed in Pubmed: 30917840.

21. Neumar RW, Shuster M, Callaway CW, et al. Part 1: Executive Summary: 2015 American Heart Association Guidelines Update for Cardiopulmonary Resuscitation and Emergency Cardiovascular Care. Circulation. 2015; 132(18 Suppl 2): S315-S367, doi: 10.1161/ CIR.0000000000000252, indexed in Pubmed: 26472989.

22. Monsieurs KG, Nolan JP, Bossaert LL, et al. ERC Guidelines 2015 Writing Group. European Resuscitation Council Guidelines for Resuscitation 2015: Section 1. Executive summary. Resuscitation. 2015; 95: 1-80, doi: 10.1016/j.resuscitation.2015.07.038, indexed in Pubmed: 26477410.

23. Solevåg AL, Schmölzer GM. Optimal Chest Compression Rate and Compression to Ventilation Ratio in Delivery Room Resuscitation: Evidence from Newborn Piglets and Neonatal Manikins. Front Pediatr. 2017; 5 : 3, doi: 10.3389/fped.2017.00003, indexed in Pubmed: 28168185.

24. Smereka J, Iskrzycki $Ł$, Makomaska-Szaroszyk E, et al. The effect of chest compression frequency on the quality of resuscitation by lifeguards. A prospective randomized crossover multicenter simulation trial. Cardiol J. 2019; 26(6): 769-776, doi: 10.5603/CJ.a2018.0121, indexed in Pubmed: 30338845.

25. Kilgannon JH, Kirchhoff M, Pierce L, et al. Association between chest compression rates and clinical outcomes following in-hospital cardiac arrest at an academic tertiary hospital. Resuscitation. 2017; 110: 154-161, doi: 10.1016/j.resuscitation.2016.09.015, indexed in Pubmed: 27666168.
26. Ewy GA, Zuercher M, Hilwig RW, et al. Improved neurological outcome with continuous chest compressions compared with 30:2 compressions-to-ventilations cardiopulmonary resuscitation in a realistic swine model of out-of-hospital cardiac arrest. Circulation. 2007; 116(22): 2525-2530, doi: 10.1161/CIRCULATIONAHA.107.711820, indexed in Pubmed: 17998457.

27. Wang S, Li C, Ji X, et al. Effect of continuous compressions and 30:2 cardiopulmonary resuscitation on global ventilation/perfusion values during resuscitation in a porcine model. Crit Care Med. 2010; 38(10): 2024-2030, doi: 10.1097/CCM.0b013e3181eed90a, indexed in Pubmed: 20683258.

28. Brown SP, Wang H, Aufderheide TP, et al. ROC Investigators. A randomized trial of continuous versus interrupted chest compressions in out-of-hospital cardiac arrest: rationale for and design of the Resuscitation Outcomes Consortium Continuous Chest Compressions Trial. Am Heart J. 2015; 169(3): 334-341.e5, doi: 10.1016/j.ahj.2014.11.011, indexed in Pubmed: 25728722.

29. Smereka J, Szarpak L, Rodríguez-Núñez A, et al. A randomized comparison of three chest compression techniques and associated hemodynamic effect during infant CPR: A randomized manikin study. Am J Emerg Med. 2017; 35(10): 1420-1425, doi: 10.1016/j. ajem.2017.04.024, indexed in Pubmed: 28433454.

30. Zhan L, Yang LiJ, Huang Yu, et al. Continuous chest compression versus interrupted chest compression for cardiopulmonary resuscitation of non-asphyxial out-of-hospital cardiac arrest. Cochrane Database Syst Rev. 2017; 3: CD010134, doi: 10.1002/14651858.CD010134.pub2, indexed in Pubmed: 28349529.

31. Liu S, Vaillancourt C, Kasaboski A, et al. Bystander fatigue and CPR quality by older bystanders: a randomized crossover trial comparing continuous chest compressions and 30:2 compressions to ventilations. CJEM. 2016; 18(6): 461-468, doi: 10.1017/cem.2016.373, indexed in Pubmed: 27650514.

32. Kovacs A, Vadeboncoeur TF, Stolz U, et al. Chest compression release velocity: Association with survival and favorable neurologic outcome after out-of-hospital cardiac arrest. Resuscitation. 2015; 92: 107-114, doi: 10.1016/j.resuscitation.2015.04.026, indexed in Pubmed: 25936931.

33. Considine J, Gazmuri RJ, Perkins GD, et al. Chest compression components (rate, depth, chest wall recoil and leaning): A scoping review. Resuscitation. 2020; 146: 188-202, doi: 10.1016/j.resuscitation.2019.08.042, indexed in Pubmed: 31536776.

34. Smereka J, Bielski K, Ladny JR, et al. Evaluation of a newly developed infant chest compression technique: A randomized crossover manikin trial. Medicine (Baltimore). 2017; 96(14): e5915, doi: 10.1097/ MD.0000000000005915, indexed in Pubmed: 28383397. 